\title{
Feature Extraction based on Sub-Pattern Multi-Directional 2DLDA
}

\author{
Xiaoqing Dong*
}

Department of Physics and Electronic Engineering, Hanshan Normal University, Chaozhou, 521041, China

\begin{abstract}
A novel feature extraction method based on sub-pattern Multi-directional two-dimensional linear discriminate analysis (Sp-MD2DLDA) for face recognition is presented in this paper. In the proposed method, firstly, we apply directional 2DLDA (D2DLDA) to extract features in some initial directions, and then choose the effective directions from the initial directions for feature fusion after an evaluation. Secondly, divide the original images into small regions and apply D2DLDA to a set of partitioned sub-patterns to obtain features in the selected effective directions which complement each other. Finally, fuse these complementary features and use nearest neighbor classifier for classification. Since the proposed method not only can extract local features and reduce the impact of the variations in expression and illumination by dividing the original images into smaller sub-images, but also extract features in many more directions, we expect that it can improve the recognition performance. The experimental results on Yale and ORL databases show that the proposed SpMD2DLDA method has better classification performance than that of the other related methods.
\end{abstract}

Keywords: Face recognition, feature extraction, feature fusion, sub-pattern multi-directional 2DLDA (Sp-MD2DLDA).

\section{INTRODUCTION}

Face recognition [1] is a hot research topic in the field of pattern recognition and image processing, and have been successfully applied to many fields. However, it is still a challenging task because the face image varies with facial expression, illumination, pose and age, noise etc. Feature extraction is the key point of face recognition [2].

The aim of feature extraction is to extract the effective features from many facial features [3]. The linear discriminate analysis (LDA) [4] is recognized as the most important feature extraction tool and still widely used in face image recognition [5, 6]. However, in these traditional methods, there are some important issues such as small sample size problem and high-dimensional image vector space by transforming the $2 \mathrm{~d}$ face image matrix into a high-dimensional image vector, which leads to be difficult to evaluate the covariance matrix accurately and results in high computational complexity to compute the corresponding eigenvectors $[3,7]$. Also, transforming the $2 \mathrm{~d}$ image matrix into $1 \mathrm{~d}$ image vectors may destroy the structural information in the image matrix.

To overcome these shortcomings, Li Ming et al. [8] and Yang Jian et al. [9] proposed a new method, named twodimensional linear discriminate analysis (2DLDA) in 2005. Instead of transforming the two-dimensional image matrix into a high-dimensional image vector, the 2DLDA directly uses the image matrix to calculate the within-class scatter matrix and the between-class scatter matrix. Thus, 2DLDA is computationally more efficient than LDA because of the lower dimensionality of the scatter matrix, and obtains better recognition performance by making full use of discriminate information among different classes [3]. For these advantages, the 2DLDA based methods have been widely used in face recognition fields in recent years [10].

Nevertheless, Noushath $\mathrm{S}$ et al. [11] indicated that 2DLDA just extracts features from the images in the row direction and ignores the correlation between the columns, and then proposed the extend 2DLDA(E2DLDA) that works in column direction to extract features from a set of training images reflecting information in each column of images. Du Haishun et al. [12] proposed a method to fuse the features extracted from these two directions. In this method, we can get two different feature spaces by applying the 2DLDA working in the row direction and E2DLDA working in the column direction respectively, and then use an effective way to fuse these two classes of features. However, it is always not sufficient for achieving high classification accuracy by extracting features in only one or two directions. In Ref. [13, 14], the authors indicated that the vectors of the image matrix in different directions have different influences for accurate classification, and proposed a method called directional two-dimensional LDA (D2DLDA) that can extract features from any direction. And then used a matching score level fusion method, named multi-directional two-dimensional LDA (MD2DLDA), to fuse several results of D2DLDA in different directions for face recognition.

However, all the methods mentioned above have a problem that they extract the most informative features all in a global manner. Consequently, some useful local information might be lost, and the variations in illumination and facial expression would make it difficult to classify. Thus some researchers propose modular based algorithms to solve this problem. Modular LDA(MLDA) [15] divided the origin images into smaller regions and considered each sub-image as a sample, and then projected all of the sub-images onto a sin- 
gle projection matrix for feature extraction. Thus, only some of the sub-images might be affected by the variations in facial expression or illumination. In Ref. [16], the authors proposed the modular two-directional $2 \mathrm{DLDA}\left(\mathrm{M}(2 \mathrm{D})^{2} \mathrm{LDA}\right)$ to reduce the computational complexity and obtain a higher recognition rate accuracy under the conditions of varying facial expression, illumination and pose. But, both the MLDA and $M(2 D)^{2}$ LDA add up all of the sub-images as a new training sample set for feature extraction, and do not use the spatial relations between the sub-images. Ref. [17] putted forward the concept of the sub-pattern which retains the spatial relations between the sub-images, and obtains a better recognition performance. However, these modular based methods only extract features in one or two directions, and some useful information in other directions might be lost.

In this paper, we combine the advantages of sub-pattern based algorithms and the MD2DLDA, and propose a new algorithm, named sub-pattern multi-directional twodimensional LDA(Sp-MD2DLDA), which is an extension of the MD2DLDA method. In the Sp-MD2DLDA method, firstly, we evaluate the recognition performance for the possible directions, since not all the features extracted from any direction are effective and some may have bad recognition performance. After the evaluation, we would get some effective directions for feature fusion. Secondly, construct the sub-pattern training sample sets in the selected directions by dividing the image matrix into small blocks. Thirdly, extract features by D2DLDA in different directions and fuse these complementary features in an effective way. Finally, classify using the nearest neighbor classifier. Compared with the traditional algorithms, the proposed method not only extracts significant features in many more directions, but also extracts more useful local features. Furthermore, variations in facial expression and illumination may affect only some of the sub-images. Hence, the proposed method is expected to have a better recognition performance. In the paper, we used two face databases to evaluate the performance of the related algorithms.

The rest of this paper is organized as follows: Section 2 describes the MD2DLDA method. Section 3 explains the proposed method sub-pattern MD2DPCA (Sp-MD2DPCA). The experiments on two public face datasets are given to compare the proposed method with several relevant methods in section 4. Finally, a conclusion is drawn in section 5.

\section{RELATED WORKS}

\subsection{DLDA}

Suppose that there are $C$ pattern classes in the training sample set and $N$ training images $(m \times n)$ in total. $N_{i}\left(i=1,2 \ldots . . C ; N=\sum_{i=1}^{C} N_{i}\right)$ denotes the number of the training samples in class $i$ and $A_{i, k}\left(k=1,2 \ldots . N_{i}\right)$ denotes the $k$ th training sample in class $i$. $\bar{A}_{i}$ and $\bar{A}$ represent the mean of the training samples in class $i$ and the mean of all training samples, respectively.

The aim of 2DLDA is to find out the optimal projection axes $x$ (an n-dimensional column vector) by using Fisher criterion which is defined as follow:
$J(x)=\frac{x^{T} G_{b-2 D L D A} x}{x^{T} G_{w-2 D L D A} x}$

Where $G_{w-2 D L D A}$ denotes the within-class scatter matrix and $G_{b-2 D L D A}$ denotes the between-class scatter matrix[3] shown as follows:

$$
\begin{aligned}
& G_{w-2 D L D A}=\frac{1}{N} \sum_{i=1}^{C} \sum_{k=1}^{N_{i}}\left(A_{i, k}-\overline{A_{i}}\right)^{T}\left(A_{i, k}-\overline{A_{i}}\right) \\
& G_{b-2 D L D A}=\frac{1}{N} \sum_{i=1}^{C} N_{i}\left(\overline{A_{i}}-\bar{A}\right)^{T}\left(\overline{A_{i}}-\bar{A}\right)
\end{aligned}
$$

In fact, the optimal projection axis is the eigenvector of $G_{b-2 D L D A} G_{w-2 D L D A}^{-1}$ corresponding to the largest eigenvalue. Because the within-class scatter matrix $G_{w-2 D L D A}$ is always nonsingular [8] in real situation, we can find out the eigenvalues and eigenvectors of $G_{b-2 D L D A} G_{w-2 D L D A}^{-1}$. In general, we need to select a set of projection axes, since only one optimal projection axes is usually not enough. In fact, these $d$ optimal projection axes are the orthonormal eigenvectors corresponding to the first $d$ largest eigenvalues, and the $X_{o p t}=\left[x_{1}, x_{2}, \ldots x_{d}\right]$ is a optimal $n \times d$ projection matrix.

For a given $\mathrm{m} \times \mathrm{n}$ image sample $A_{i, k}$, let

$Y=A_{i, k} X \quad\left(k=1,2, \ldots . N_{i}\right)$

where $Y=\left[Y_{1}, Y_{2}, \ldots Y_{d}\right]$ is the $\mathrm{m} \times \mathrm{d}$ feature matrix of the image sample $A_{i, k}$.

\subsection{D2DLDA}

We can obtain a rotated image in a certain direction of $\alpha$-degree, denoted as $r_{\alpha}\left(A_{i, k}\right)$, by using original image multiplying the rotation matrix $R(\alpha)$ shown as eq.(5) in Euclidean space[13,14].

$R(\alpha)=\left[\begin{array}{cc}\cos \alpha & -\sin \alpha \\ \sin \alpha & \cos \alpha\end{array}\right]$

Fig. (1) shows an image rotated in the directions of $\alpha=\frac{\pi}{4}$ and $\alpha=\frac{\pi}{2}$.

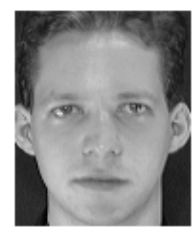

(a)

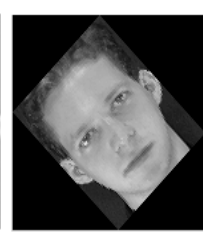

(b)

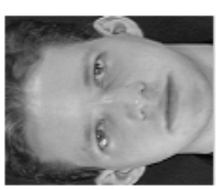

(c)
Fig. (1). (a) The Original Image. (b) The Image Rotated with $\alpha=\frac{\pi}{4}$. (c) The Image Rotated with $\alpha=\frac{\pi}{2}$.

By employing 2DLDA to the rotated images, we can extract features in the direction in which the origin image rotated. Since the image can be rotated in any direction, we can 


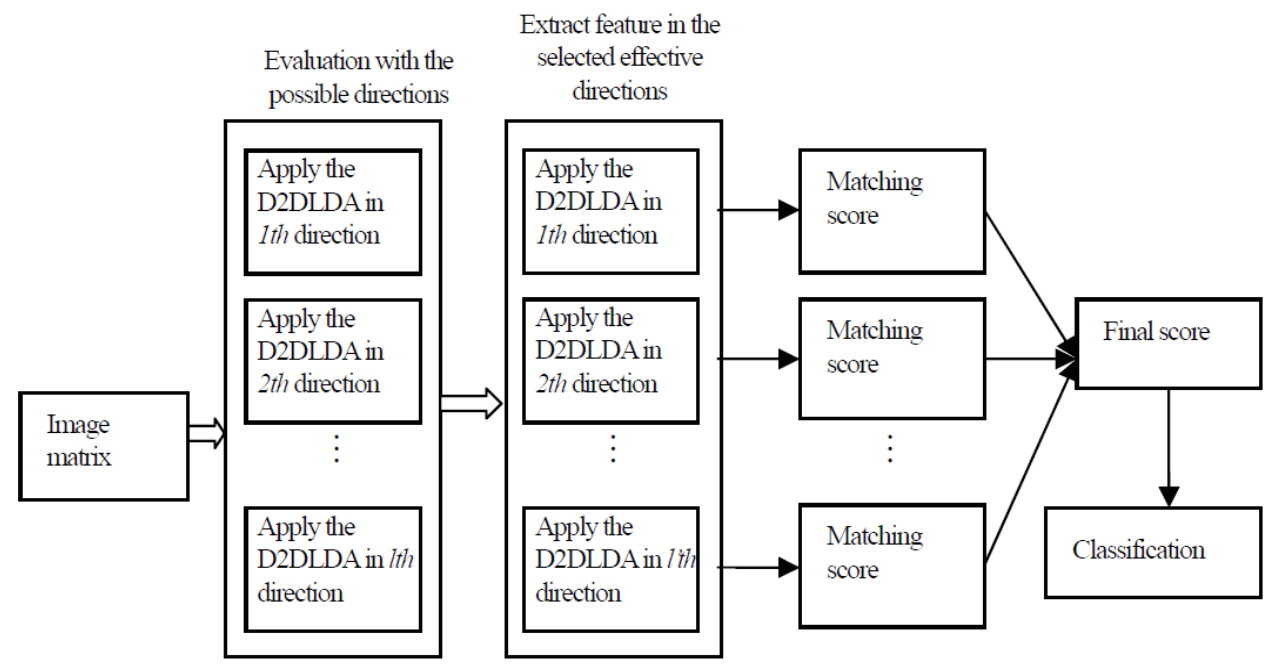

Fig. (2). The framework of MD2DLDA.

extract features in any direction, and this method was named directional 2DLDA(D2DLDA) [14].

Similarly to 2DLDA, the aim of D2DLDA is to find out the optimal projection matrix $\mathrm{X}_{\mathrm{opt}}^{D 2 D L D A}$ by using Fisher criterion which is defined as follow:

$J(x)=\frac{x^{T} G_{b-D 2 D L D A} x}{x^{T} G_{w-D 2 D L D A} x}$

where $G_{b-D 2 D L D A}$ and $G_{w-D 2 D L D A}$ denote the between-class scatter matrix and the within-class scatter matrix shown as follows:

$$
\begin{aligned}
G_{b-D 2 D L D A} & =\frac{1}{N} \sum_{i=1}^{C} N_{i}\left(\overline{r_{\alpha}\left(A_{i}\right)}-\overline{r_{\alpha}(A)}\right)^{T}\left(\overline{r_{\alpha}\left(A_{i}\right)_{i}}-\overline{r_{\alpha}(A)}\right) \\
G_{w-D 2 D L D A} & =\frac{1}{N} \sum_{i=1}^{C} \sum_{k=1}^{N_{i}}\left(r_{\alpha}\left(A_{i, k}\right)-\overline{r_{\alpha}\left(A_{i}\right)}\right)^{T}\left(r_{\alpha}\left(A_{i, k}\right)-\overline{r_{\alpha}\left(A_{i}\right)}\right)
\end{aligned}
$$

where $r_{\alpha}\left(A_{i, k}\right)\left(k=1,2 \ldots . N_{i}\right)$ represents the $k t h$ rotated training image in class $i . \overline{r_{\alpha}(A)}$ and $\overline{r_{\alpha}\left(A_{i}\right)}$ denote the mean of all the rotated training samples and the mean of the rotated training samples in class $i$, respectively.

Calculate the eigenvalues and eigenvectors of the $G_{b-D 2 D L D A} G_{w-D 2 D L D A}^{-1}$, and select $d$ orthonormal eigenvectors corresponding to the $d$ largest eigenvalues as projection axes, i.e. $\quad X_{1}^{D 2 D L D A}, X_{2}{ }^{D 2 D L D A}, \ldots X_{d}{ }^{D 2 D L D A}$. Hence the $X_{\text {opt }}^{D 2 D L D A}=\left[x_{1}^{D 2 D L D A}, x_{2}{ }^{\text {D2DLDA }}, \ldots x_{d}^{\text {D2DLDA }}\right]$ is the optimal $n \times d$ projection matrix.

\subsection{MD2DLDA}

In Ref. [13, 14], the authors indicated that using the complementary features extracted in different directions simultaneously is helpful for increasing recognition rate, and then proposed an effective fusion method named multidirectional 2DLDA(MD2DLDA) to fuse these complementary features extracted by D2DLDA in different directions for classification. Since not all the features which are extracted in different directions are effective, we must evaluate the recognition performance for the possible directions before feature fusion, and only select the effective directions for feature fusion. The flowchart of MD2DLDA is shown in Fig. (2) and its process is implemented as follows [14]:

Step 1. Evaluate the recognition performance of the possible directions. Firstly, rotate the training images in the possible $l$ directions(such as 0 -degree, 30-degree, 60-degree, 90degree, 120-degree, 150-degree, 36-degree, 72-degree, 108degree, 144-degree,45-degree and 135-degree), which are derived from the 180-degree dividing by $3,4,5$ and 6 , respectively. Secondly, perform D2DLDA in each direction to obtain the projection matrixes and use the nearest neighbor classifier for classifying. Finally, choose the effective $l$ ' directions for feature fusion $\left(\left(l^{\prime}<l\right)\right)$.

Step 2. Features extraction: Perform D2DLDA in all the selected effective directions to obtain the projection matrixes, then extract the feature matrixes of the training samples and the testing samples by using Eq.(4).

Step 3. Features fusion. First, calculate the matching score between each testing sample and training sample which are all rotated in the same direction of $\alpha$-degree using:

$s_{\alpha, i}=\sum_{p=1}^{d}\left\|Y_{i}^{p}-Y^{p}\right\|(i=1,2, \cdots, N)$

where $Y_{i}^{p}$ and $Y^{p}$ denote the $p t h$ eigenvector of the feature matrix of training sample $A_{i}$, and testing sample $A$, respectively. Hence $\left\|Y_{i}^{p}-Y^{p}\right\|$ is the Euclidean distance between these two vectors and $s_{\alpha, i}$ is the Euclidean distance between their feature matrixes.

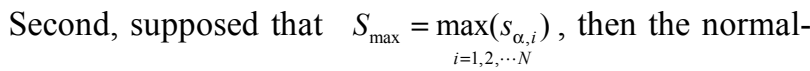
ized distance can be defined as

$s_{\alpha, i}^{\prime}=s_{\alpha, i} / S_{\max }(i=1,2, \cdots, N)$

The final matching score of feature fusion in the selected $l$ ' '( $l$ ' $\leq l$ ') effective directions can be calculated by: 
$s_{i}=\sum_{\alpha=1 \text { th-direction }}^{l^{\prime \prime} \text { th-direction }} s_{\alpha, i}^{\prime}(i=1,2, \cdots, N)$

where $\alpha=1$ th-direction and $l "$ th-direction denote the 1 th and $l$ ' $t h$ selected directions which are selected in step 1 . The fusion directions are selected from all the effective $l$ ' directions, so $l$ ' ' is less than or equal to $l$ '.

Step4. Classification: Apply the final matching scores to classify by using the nearest neighbor classifier.

\section{SUB-PATTERN MULTI-DIRECTIONAL LDA: SP- MD2DLDA}

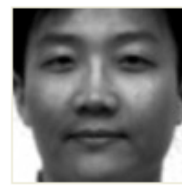

(a)

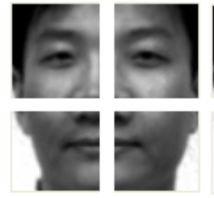

(e)

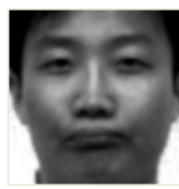

(b)

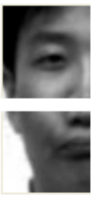

(f)

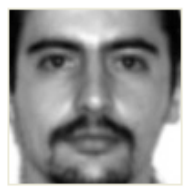

(c)

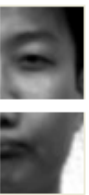

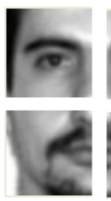

(g)

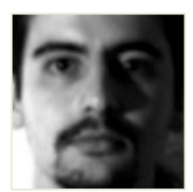

(d)

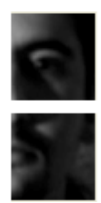

(h)
Fig. (3). Samples from the yale database. (a) and (c) Normal image. (b) Variation in facial expression. (d) Variation in illumination. (eh) Sub-images by dividing the (a-d) into four blocks, respectively.

As shown in Fig. (3), under the conditions of varying facial expression or illumination shown as image (b) and (d), the un-modular methods(including 2DLDA, (2D)2LDA, MD2DLDA) may be not very effective, since they only consider the global information of the images, and the features extracted from them would vary considerably from the features of the images with normal facial expression shown as image (a) and (c) [18]. If the face images are divided into small regions as images (e) (h), only some of the subimages are affected by varying facial expression and illumination, and rest of the sub-images will remain the same. For example, the lower part of image (b) is affected by varying facial expression, hence it is difficult for the un-modular methods to identify them correctly. But, if we divide image (b) into small regions shown as image (f), we know that only the two sub-images in lower part are affected by varying facial expression, and the two sub-images not affected by varying facial expression in upper part closely match with the upper part of the same person under normal conditions which is shown as image (e). In the similar way, the variation in illumination would only affect the two sub-images in right half of image (h), and the two sub-images in left half closely match with the left half of the same person under normal conditions as shown in image $(\mathrm{g})$. Therefore, it is expected that the proposed method Sp-MD2DLDA would have better recognition performance under the conditions of varying facial expression and illumination, since it not only extracts more informative features from local regions and different directions, but also reduces the influence of the variations in facial expression and illumination by dividing the images into small blocks.

In this section, we present the details of the proposed $\mathrm{Sp}$ MD2DLDA method. The flowchart of Sp-MD2DLDA is shown in Fig. (4).

As shown in Fig. (4), the process of the Sp-MD2DLDA is implemented by the following steps:

Step1. Evaluate the recognition performance of the possible directions. Similar to the step 1 of MD2DLDA, first, we rotate the training images in the possible $l$ directions (such as 0-degree, 30-degree, 60-degree, 90-degree, 120degree, 150-degree,36-degree,72-degree, 108-degree, 144degree,45-degree and 135-degree), which are derived from the 180-degree dividing by 3,4,5 and 6 , respectively. Let image $A$ be an m by $\mathrm{n}$ image matrix and $A_{i, k}$ denotes the $k t h$ sample image in class $i$, then the image matrix in the $\alpha$ direction can be denoted as follow:

$$
r_{\alpha}\left(A_{i, k}\right)=\left[\begin{array}{cccc}
r_{\alpha}\left(A_{i, k}\right)_{11} & r_{\alpha}\left(A_{i, k}\right)_{12} & \cdots & r_{\alpha}\left(A_{i, k}\right)_{1 n} \\
r_{\alpha}\left(A_{i, k}\right)_{21} & r_{\alpha}\left(A_{i, k}\right)_{22} & \cdots & r_{\alpha}\left(A_{i, k}\right)_{2 n} \\
\vdots & \vdots & \vdots & \vdots \\
r_{\alpha}\left(A_{i, k}\right)_{m 1} & r_{\alpha}\left(A_{i, k}\right)_{m 2} & \cdots & r_{\alpha}\left(A_{i, k}\right)_{m n}
\end{array}\right]
$$

The new position of each pixel in the rotated image matrix is obtained by using original position multiplying the rotation matrix $R(\alpha)$ (Eq.(5)) in Euclidean space[16].

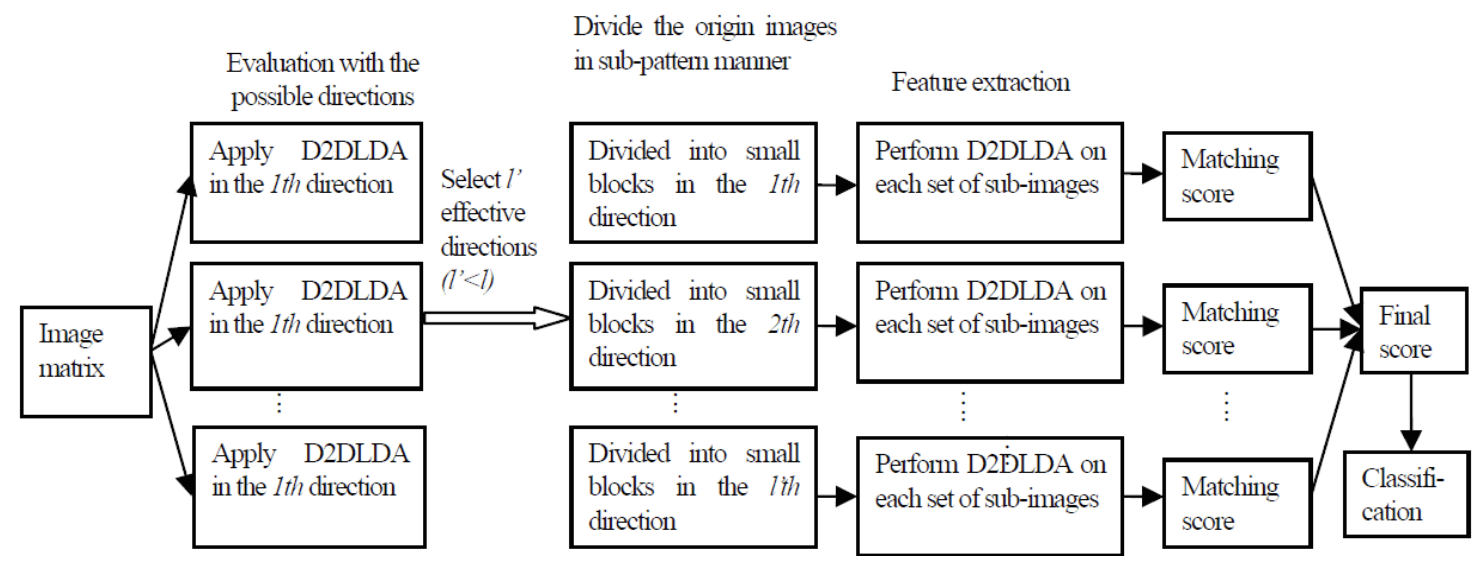

Fig. (4). The flowchart of Sp-MD2DLDA. 
Second, perform D2DLDA in each direction to obtain the projection matrixes and use the nearest neighbor classifier for classifying. Finally, choose the effective $l$ ' directions for feature fusion $\left(\left(l^{\prime}<l\right)\right)$.

Step2. Construct the sub-pattern training sample sets in the selected directions. Divide the image matrix of $A_{i, k}$ into $N=p^{*} q$ sub-images, where $p$ and $q$ are the numbers of the blocks in vertical and horizontal directions, as given in (13).

$$
r_{\alpha}\left(A_{i, k}\right)=\left[\begin{array}{cccc}
r_{\alpha}\left(B_{i, k}\right)_{11} & r_{\alpha}\left(B_{i, k}\right)_{12} & \cdots & r_{\alpha}\left(B_{i, k}\right)_{1 q} \\
r_{\alpha}\left(B_{i, k}\right)_{21} & r_{\alpha}\left(B_{i, k}\right)_{22} & \cdots & r_{\alpha}\left(B_{i, k}\right)_{2 q} \\
\vdots & \vdots & \vdots & \vdots \\
r_{\alpha}\left(B_{i, k}\right)_{p 1} & r_{\alpha}\left(B_{i, k}\right)_{p 2} & \cdots & r_{\alpha}\left(B_{i, k}\right)_{p q}
\end{array}\right]
$$

where $r_{\alpha}\left(B_{i, k}\right)_{x y}$ denotes each sub-image of the rotated image matrix. The width and height of each sub-image can be calculated as $m_{1}=m / p$ and $n_{1}=n / q$, respectively. Suppose that there are $N$ training image samples in total. Let all the sub-images of the same position as a new sub-pattern training sample set, and the sample size of each set is also $N$. Now we have $p^{*} q$ sub-pattern training sample sets $T_{x y}$ in a direction of $\alpha$-degree.

It is expected that the image divided into smaller subimages is helpful for extracting more local information and reducing the influence of varying facial expression and illumination. Nevertheless, the face images can not be divided into very small regions, because most of the global information of the face may be lost. The optimal block size ultimately depends on the characteristics of each particular database [17].

Step3. Feature extraction by projecting the samples onto their corresponding projection matrixes.

The image between-class scatter matrix $r_{\alpha}\left(G_{b-2 D L D A}\right)_{x y}$ and within-class scatter matrix $r_{\alpha}\left(G_{w-2 D L D A}\right)_{x y}[12]$ of $T_{x y}$ are defined as follows:

$$
\begin{aligned}
r_{\alpha}\left(G_{b-2 D L D A}\right)_{x y}= & \frac{1}{N} \sum_{i=1}^{C} N_{i}\left(\overline{r_{\alpha}\left(B_{i}\right)_{x y}}-\overline{r_{\alpha}(B)_{x y}}\right)^{T} \\
& \left(\overline{r_{\alpha}\left(B_{i}\right)_{x y}}-\overline{r_{\alpha}(B)_{x y}}\right) \\
r_{\alpha}\left(G_{w-2 D L D A}\right)_{x y}= & \frac{1}{N} \sum_{i=1}^{C} \sum_{k=1}^{N_{i}}\left(r_{\alpha}\left(B_{i, k}\right)_{x y}-\overline{r_{\alpha}\left(B_{i}\right)_{x y}}\right)^{\mathrm{T}} \\
& \left(r_{\alpha}\left(B_{i, k}\right)_{x y}-\overline{r_{\alpha}\left(B_{i}\right)_{x y}}\right)
\end{aligned}
$$

where $\overline{r_{\alpha}(B)_{x y}}, \overline{r_{\alpha}\left(B_{i}\right)_{x y}}$ denote the mean of all training samples of $T_{x y}$ and the mean of the training samples in class $i$ of $T_{x y}$, respectively. Apply the Fisher criterion to all the subpattern training sample sets, and select the orthonormal eigenvectors of $r_{\alpha}\left(G_{b-2 D L D A}\right)_{x y} / r_{\alpha}\left(G_{w-2 D L D A}\right)_{x y}$ corresponding to the first $d$ largest eigenvalues as the optimal projection matrix, which is an $n \times d$ matrix as $r_{\alpha}(X)_{x y}=\left[r_{\alpha}\left(x_{1}\right)_{x y}, r_{\alpha}\left(x_{2}\right)_{x y}, \ldots r_{\alpha}\left(x_{d}\right)_{x y}\right]$.

According to Eq.(4), the feature matrix of sub-image $r_{\alpha}\left(B_{i, k}\right)_{x y}$ can be obtained by $\left(B_{i, k}\right)_{x y} X_{x y}$, which size is $m 1 \times d$. Since the feature matrix of the origin image $r_{\alpha}\left(A_{i, k}\right)$ consists of feature matrixes of all the sub-images, it can be denoted as

$$
r_{\alpha}\left(Y_{i, k}\right)=\left[\begin{array}{cccc}
r_{\alpha}\left(B_{i, k}\right)_{11} X_{11}^{T} & r_{\alpha}\left(B_{i, k}\right)_{12} X_{12}^{T} & \cdots & r_{\alpha}\left(B_{i, k}\right)_{1 q} X_{1 q}^{T} \\
r_{\alpha}\left(B_{i, k}\right)_{21} X_{21}^{T} & r_{\alpha}\left(B_{i, k}\right)_{22} X_{22}^{T} & \cdots & r_{\alpha}\left(B_{i, k}\right)_{2 q} X_{2 q}^{T} \\
\vdots & \vdots & \vdots & \vdots \\
r_{\alpha}\left(B_{i, k}\right)_{p 1} X_{p 1}^{T} & r_{\alpha}\left(B_{i, k}\right)_{p 2} X_{p 2}^{T} & \cdots & r_{\alpha}\left(B_{i, k}\right)_{p q} X_{p q}^{T}
\end{array}\right](16)
$$

The size of $r_{\alpha}\left(Y_{i, k}\right)$ is $m \times q d$. In the same way, we can get the feature matrix of the test sample $r_{\alpha}\left(A_{t}\right)$ which is shown as follow:

$$
r_{\alpha}\left(Y_{t}\right)=\left[\begin{array}{cccc}
r_{\alpha}\left(B_{t}\right)_{11} X_{11}^{T} & r_{\alpha}\left(B_{t}\right)_{12} X_{12}^{T} & \cdots & r_{\alpha}\left(B_{t}\right)_{1 q} X_{1 q}^{T} \\
r_{\alpha}\left(B_{t}\right)_{21} X_{21}^{T} & r_{\alpha}\left(B_{t}\right)_{22} X_{22}^{T} & \cdots & r_{\alpha}\left(B_{t}\right)_{2 q} X_{2 q}^{T} \\
\vdots & \vdots & \vdots & \vdots \\
r_{\alpha}\left(B_{t}\right)_{p 1} X_{p 1}^{T} & r_{\alpha}\left(B_{t}\right)_{p 2} X_{p 2}^{T} & \cdots & r_{\alpha}\left(B_{t}\right)_{p q} X_{p q}^{T}
\end{array}\right]
$$

Step 4. Feature fusion. After obtaining the feature matrixes in all the selected directions, we calculate the Euclidean distance between each testing sample and training sample by using eq.(18), similarly to MD2DLDA in section2.3.

$r_{\alpha}\left(D_{i, k}\right)=\sum_{j=1}^{q d}\left\|r_{\alpha}\left(Y_{i, k}^{j}\right)-r_{\alpha}\left(Y_{t}^{j}\right)\right\|$

where $r_{\alpha}\left(Y_{i, k}^{j}\right)$ and $r_{\alpha}\left(Y_{t}^{j}\right)$ represent the $j t h$ feature vector of the feature matrix $r_{\alpha}\left(Y_{i, k}\right)$ and $r_{\alpha}\left(Y_{t}\right)$, respectively. $\left\|r_{\alpha}\left(Y_{i, k}^{j}\right)-r_{\alpha}\left(Y_{t}^{j}\right)\right\|$ Denotes the Euclidean distance between $r_{\alpha}\left(Y_{i, k}^{j}\right)$ and $r_{\alpha}\left(Y_{t}^{j}\right)$.

To fuse the features extracted in different directions, suppose that $r_{\alpha}\left(D_{\max }\right)=\max \left(r_{\alpha}\left(D_{i, k}\right)\right)$, then the normalized distance can be denoted as:

$$
r_{\alpha}\left(D_{i, k}\right)^{\prime}=r_{\alpha}\left(D_{i, k}\right) / r_{\alpha}\left(D_{\max }\right)
$$

So the final distance between the testing sample and training sample in different directions can be defined as follow:

$$
D_{i, k}=\sum_{\alpha=1 t h-\text { direction }}^{l " t h-\text { direction }} r_{\alpha}\left(D_{i, k}\right)^{\prime}
$$

where $\alpha=1$ th-direction and $l " t h$-direction denote the lth and $l " t h$ directions which are selected in step 1 . Since the directions used for feature fusion are selected from the effective $l$ ' directions, $l$ ' 'is no more than $l$ '.

Step 5. Based on Eq. (18), Eq. (19) and Eq. (20), calculate all the final distance between the testing sample and training samples, then classify using the nearest neighbor classifier.

\section{EXPERIMENTAL RESULTS}

In the experiments, we compared the proposed method with the 2DLDA, (2D) ${ }^{2}$ LDA, MD2DLDA, M2DLDA and 
$\mathrm{M}(2 \mathrm{D})^{2} \mathrm{LDA}$ methods on two image databases, Yale and ORL databases. The Yale database consists of images with varying illumination and expressions, and was used to test the performance of the system for varying the number of eigenvectors. The ORL database contains images with varying facial expression and pose, and was employed to examine the performance of the related algorithms for varying number of sample size. We used MATLAB2010 for all of the experiments and performed on a $\mathrm{PC}$ with Pentium(R) Dual-Core E5800 3.20GHz CPU, 2GB memory and windows XP operating system.

\subsection{Experiments on Yale Face Database}

The Yale database has 165 images of 15 persons(each person has 11 images). The face images are under various facial expressions (including normal, sad, happy and surprised expressions). There are also images with and without glasses. In addition to these, there are images where the position of the light source is from different directions. Each image was manually cropped and resized to $100 * 100$ pixels in this experiment. Fig. (5) shows the images of a person from the Yale database.
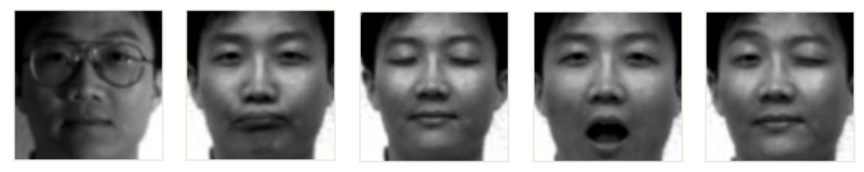

Fig. (5). Five images of one person from the yale face database.

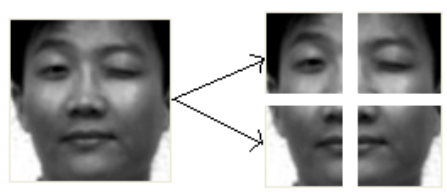

Fig. (6). A face image divided into $2 * 2$ smaller images.

In these experiments, we tested the performance of the related methods with different number of eigenvectors (from 1 to 20). We selected 6 images from each person as training samples, and the remaining 5 images are as testing samples. Thus, the total number of training samples and testing samples are 90 and 75, respectively. In order to eliminate the randomness of the sample selection, 20 possible selections of training samples were randomly chosen in the experiments, and the experiments were repeated independently 20 times with these selections. Then we took the average recognition rates as the final recognition rates. In the experiments, we used the case of the $2 * 2$ (denotes as $k=4$ ) blocks for the modular based methods (including M2DLDA, M(2D) ${ }^{2}$ LDA and the proposed Sp-MD2DLDA), as shown in Fig. (6).

According to the process of the proposed Sp-MD2DLDA which is mentioned in section 3 , firstly, we must evaluate the recognition performance in different directions to get some effective directions for the further processing. Then, divide the original images into smaller sub-images and apply D2DLDA to a set of partitioned sub-patterns to obtain features in the selected effective directions. Finally, fuse the features extracted from the effective directions according to the Eq.(18), Eq.(19) and Eq.(20), and used the nearest neighbor classifier for classification.

\section{(1). Evaluation for Possible Directions}

Based on the D2DLDA, the images were rotated in the following directions(degrees): 0,30 , $60,90,120,150,180,36,72,108,144,45$ and 135 degrees. These directions were derived from the 180-degree dividing by $3,4,5$ and 6 , respectively. The 2DLDA and E2DLDA can be seen as the D2DLDA in 0-degree and 90-degree directions respectively, and the images rotated in 0 -degree is equal to the images rotated in 180-degree. We tested the performance of the D2DLDA for varying number of eigenvectors (from 1 to 20 ).

Fig. (7) shows the results of the D2DLDA under the condition of varying number of eigenvectors. It can be observed from Fig. (7) that the recognition rates are increasing in all directions as we increase the number of eigenvectors(denotes as $\mathrm{E}$ ) when $\mathrm{E}<5$, and tend to be stable when $\mathrm{E}>5$. The proposed method in the directions of 0 -degree, 30-degree, 60degree, 90-degree, 120-degree and 150-degree obtains significantly higher recognition rates(particularly in the directions of 0 -degree, 30-degree, 90-degree and 150-degree ) than that in the directions of 36-degree, 45-degree, 72degree, 108-degree, 135-degree and 144-degree. So, we only selected the directions of 0 -degree, 30-degree, 90-degree and 150 -degree for feature fusion.

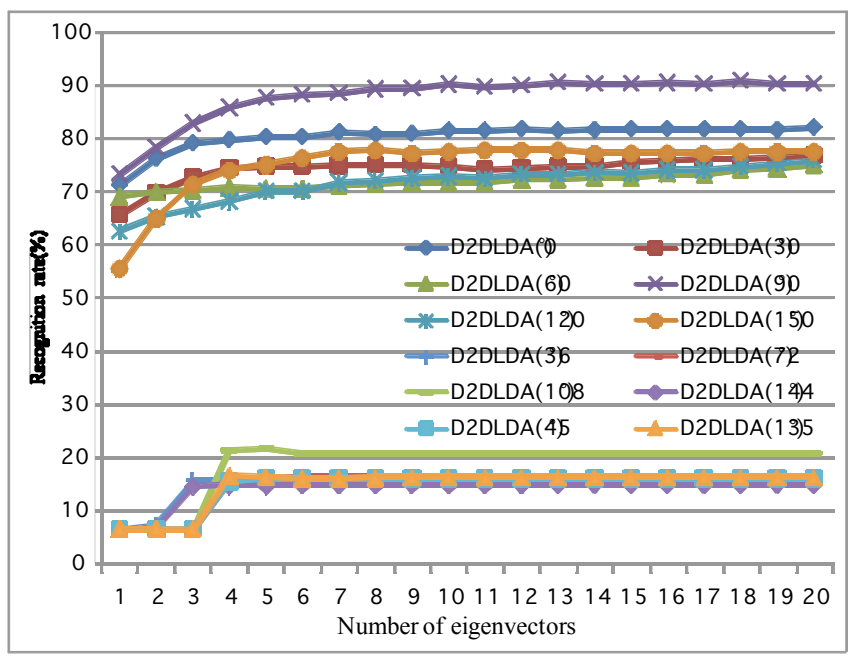

Fig. (7). Recognition rates of $\mathrm{d} 2 \mathrm{~d}$ lda under the condition of varying number of eigenvectors.

\section{(2). Features Fusion in The Selected Directions}

From the evaluation results shown in Fig. (7), we note that the proposed method in the directions of 0 -degree, 30 degree, 90-degree and 150-degree has better performance than the other directions, so we selected these directions for feature fusion. There are many possible combinations for features fusion in different directions, such as the fusions in the directions of 0 -degree and 30-degree, 0 -degree and 90 degree, etc.

As shown in Table 1, for example, " $0+30$ "denotes that the features used to be fused are in the directions of 0-degree and 30 -degree. The total number of fusion ways for two different directions, three different directions and four different directions are 6,4 and 1 , respectively. In the experiments, we tested the proposed method in all the fusion ways and just 
Table 1. All the possible combinations of feature fusion in different directions.

\begin{tabular}{|c|c|c|}
\hline 2 Different Directions & 3 Different Directions & 4 Different Directions \\
\hline \hline $0+30$ & $0+30+90$ & $0+30+90+150$ \\
\hline $0+90$ & $0+30+150$ & \\
\hline $0+150$ & $0+90+150$ & \\
\hline $30+90$ & $30+90+150$ & \\
\hline $30+150$ & & \\
\hline $90+150$ & & \\
\hline
\end{tabular}

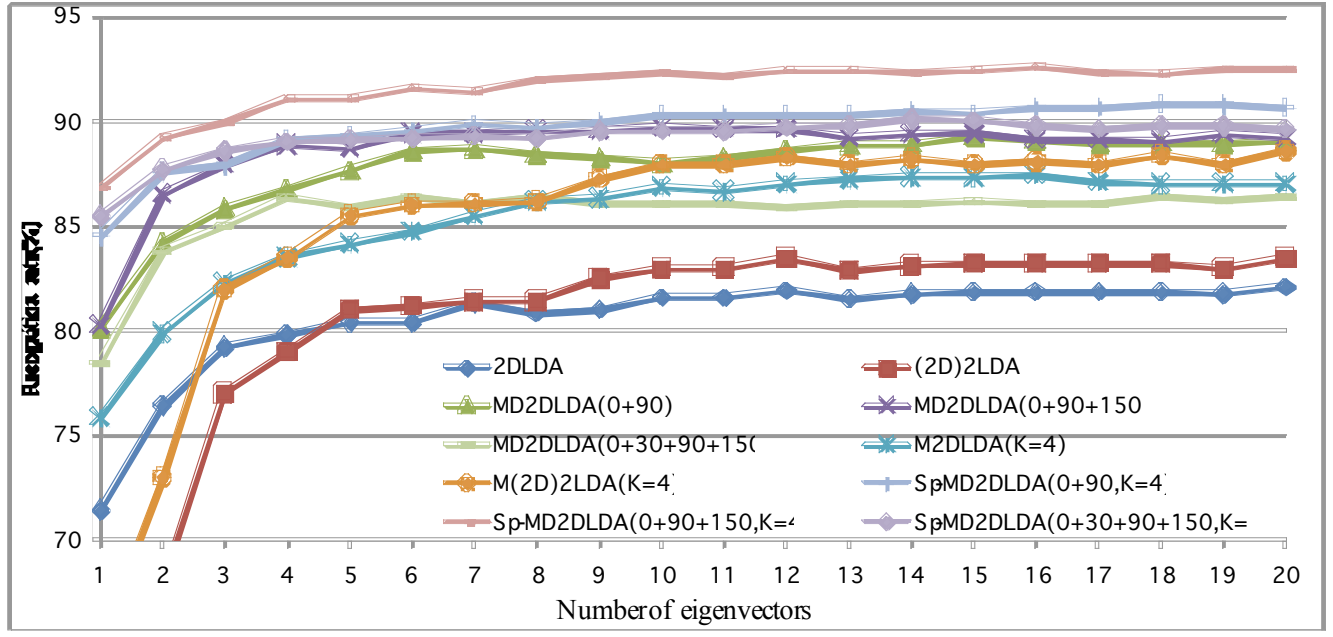

Fig. (8). Recognition rates of related methods with varying number of eigenvectors.

chosen one of the best way in each different number of directions to compare with the related method. In the experiment, the proposed Sp-MD2DLDA in the fusion directions of $0+90,0+90+150$ and $0+30+90+150$ obtain the best performance in 2,3 and 4 fusion directions, respectively.

The Fig. (8) shows the recognition rates of the related methods under the condition of varying number of eigenvectors (from 1 to 20). We can observe from the Fig. (8) that the recognition rates are increasing for all the methods as we increase the number of eigenvectors when $\mathrm{E}<5$, and the recognition rates tend to be stable when $E \geq 5$. From the comparison results of M2DLDA and 2DLDA, M(2D) ${ }^{2} \mathrm{LDA}$ and (2D) ${ }^{2}$ LDA,Sp-MD2DLDA and MD2DLDA, we know that the modular based methods(including M2DLDA, $\mathrm{M}(2 \mathrm{D})^{2} \mathrm{LDA}$ and Sp-MD2DLDA) have higher recognition rates than the un-modular based methods(including 2DLDA, (2D) ${ }^{2}$ LDA and MD2DLDA), Since the modular based methods can effectively extract local features and reduce the influence of variations in illumination and expression by dividing the origin images into smaller sub-images. In the three un-modular based methods, the MD2DLDA has the best performance than the other two methods, because the MD2DLDA can fuse the complementary features extracted in many more directions, and the 2DLDA only extracts features in the row direction and the $(2 \mathrm{D})^{2} \mathrm{LDA}$ is easy to lose some useful discriminate information by compressing in the row and column directions at the same time. In all different numbers of eigenvectors, the proposed Sp-MD2DLDA (particularly in the fusion directions of $0+90+150$ ) always obtains the highest recognition rates, since it not only fuses the complementary features extracted in many more directions, but also effectively extracts local features from the images and reduces the influence of variations in illumination and expression by dividing the origin images into smaller subimages.

Fig. (9) shows the recognition rates of the proposed method for varying the number of sub-images(denotes as $K$ ) from $2(2 * 1)$ to $16(4 * 4)$ to observe the effect of $K$ on face recognition. From the results, we note that the proposed SpMD2DLDA has the best performance in the case of $K=8$, and recognition rates declines when $K=16$, because the global information may be lost by dividing the origin images into very small regions.

\subsection{Experiments on ORL Face Database}

The ORL database includes 400 gray-scale images of 40 individuals, each providing 10 different images. The images of each subject vary with facial expressions, facial details, scale and limited rotation. Moreover, there is also some tilting and rotation of the face of up to 20 degrees. All images are normalized to a resolution of $112 * 92$ pixels. Five sample images of one subject in the ORL database are shown in Fig. (10). 


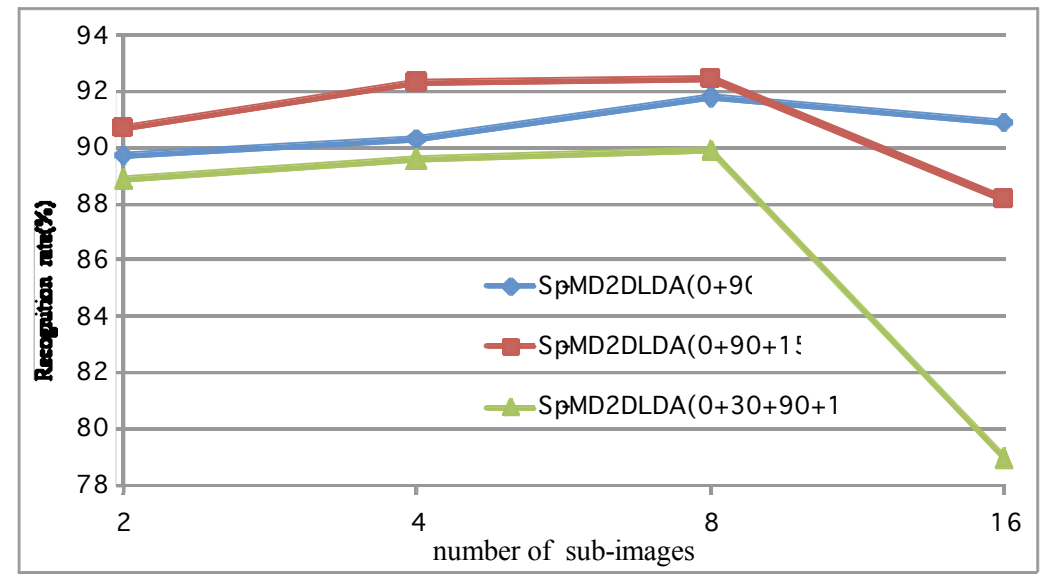

Fig. (9). Recognition rates of the proposed sp-md2dlda with varying number of sub-images.
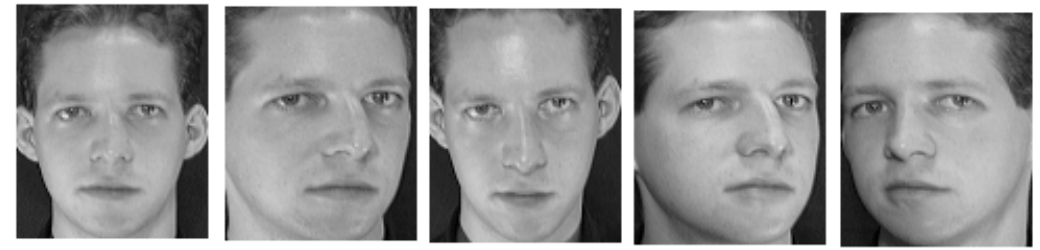

Fig. (10). Five images of one person from orl face database.

In the experiments, we tested the related methods for varying the number of the training samples(denotes as $\mathbf{S}$ ) from $2 \sim 8$ to observe the effect of sample size on recognition performance, and the remaining 10-S images were used as the testing samples. Thus, the total number of training samples and testing samples are $S * 40$ and $(10-S) * 40$, respectively. Similar to the experiments on Yale database, 20 possible selections of training images were randomly chosen in the experiments to eliminate the randomness of the sample selection, and the experiments were independently repeated 20 times with these selections. Then we took the average recognition rates as the final recognition rates. In the experiments, we used the case of the $2 * 2$ blocks $(K=4)$ for the modular based methods(including M2DLDA, M(2D) ${ }^{2}$ LDA and the proposed Sp-MD2DLDA), and only the first 10 eigenvectors are used in the experiments, since varying $\mathrm{E}$ would have the same effect on the algorithms.

Similar to the experiments on Yale database, according to the process of Sp-MD2DLDA mentioned in section 3, firstly, we must evaluate the D2DLDA in different directions to get some effective directions for subsequent processing. Secondly, divide the original images into small regions and apply D2DLDA to a set of partitioned sub-patterns to obtain features in the selected effective directions. Finally, use a fusion method to fuse the features extracted from the effective directions according to the Eq.(18), Eq.(19) and Eq.(20), and use the nearest neighbor classifier for classification.

\section{(1). Evaluation for Possible Directions}

Similar to section 4.1.1, based on the D2DLDA, the images were rotated to the following directions(degrees): 0,30 , $60,90,120,150,180,36,72,108,144,45$ and 135 degrees, which were derived from the 180 -degree dividing by $3,4,5$ and 6, respectively. The 2DLDA and E2DLDA can be seen as the special cases of D2DLDA in 0-degree and 90-degree directions respectively, and the images in the direction of 0 degree is equal to the images in the direction of 180-degree. We tested the performance of the D2DLDA in the above directions for varying number of training samples (from 2 to 8 ).

Fig. (11) shows the results of the D2DLDA with varying number of sample size. We can observe from Fig. (11) that the D2DLDA in the directions of 0-degree, 90-degree, 45degree and 135-degree (particularly the directions of 0 degree and 90-degree) has significantly higher recognition rates than that of other directions. And the directions of 30degree, 60-degree, 120-degree, 150-degree, 36-degree, 72 degree, 108-degree and 144-degree are not so effective that their recognition rates are all below $20 \%$. Therefore, we selected the directions of 0-degree, 45-degree, 90-degree and 135-degree for feature fusion.

\section{(2). Features Fusion in The Selected Directions}

After the evaluation, we note that the proposed method in the directions of 0 -degree, 45-degree, 90-degree and 135degree has better performance than the other directions, so we selected these directions for feature fusion. Table 2 shows all the possible combinations for features fusion in different directions, such as the fusions in the directions of 0 degree and 45-degree(denotes as $0+45$ ), 0 -degree and 90 degree, etc.

As shown in Table 2, the total number of combinations for two different directions, three different directions and four different directions are 6,4 and 1 , respectively. In the experiments, we tested the proposed method in all the fusion ways and only chosen the best combinations in each different number of directions to compare with the related method. In the experiments, the proposed Sp-MD2DLDA in the fusion directions of $0+90,0+90+135$ and $0+45+90+135$ obtains the best performance in 2,3 and 4 fusion directions, respectively. 


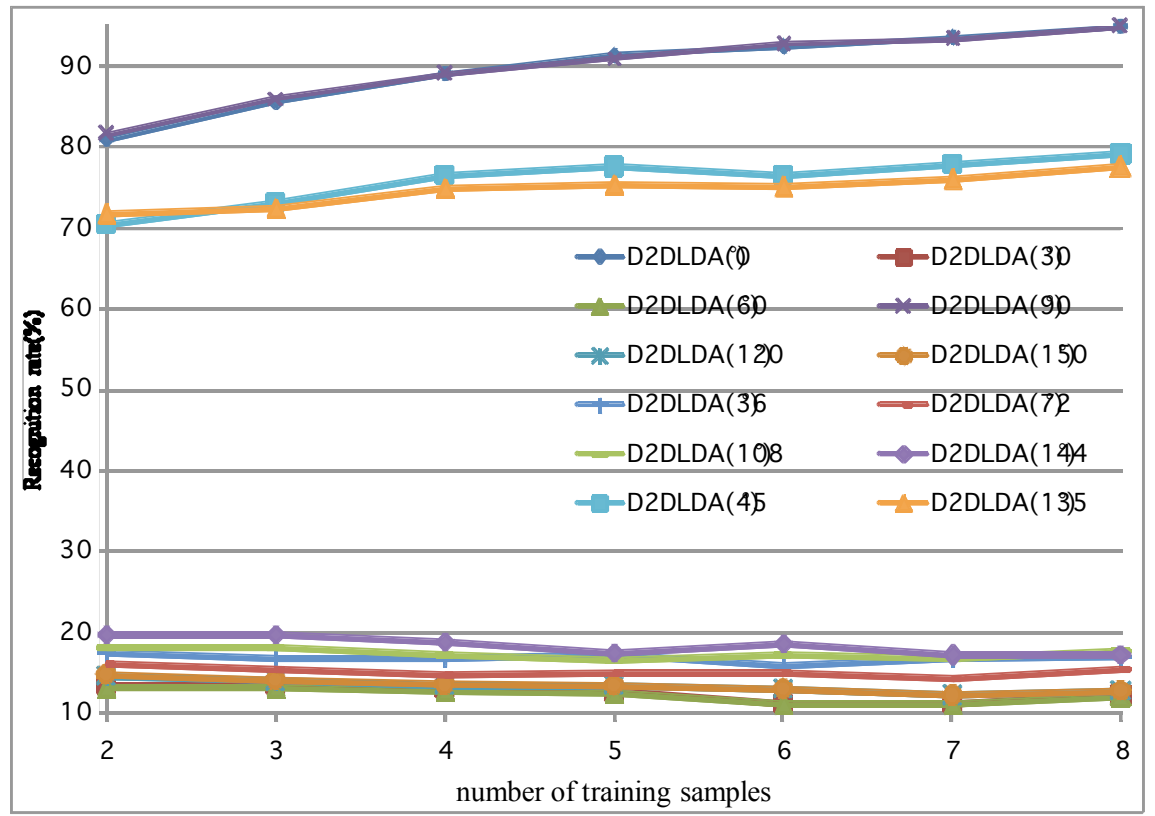

Fig. (11). Recognition rates of D2DLDA by varying number of sample size.

Table 2. All the possible combinations of feature fusion in different directions.

\begin{tabular}{|c|c|c|}
\hline 2 Different Directions & 3 Different Directions & 4 Different Directions \\
\hline \hline $0+45$ & $0+45+90$ & $0+45+90+135$ \\
\hline $0+90$ & $0+45+135$ & \\
\hline $0+135$ & $0+90+135$ & \\
\hline $45+90$ & $45+90+135$ & \\
\hline $45+135$ & & \\
\hline $90+135$ & & \\
\hline
\end{tabular}

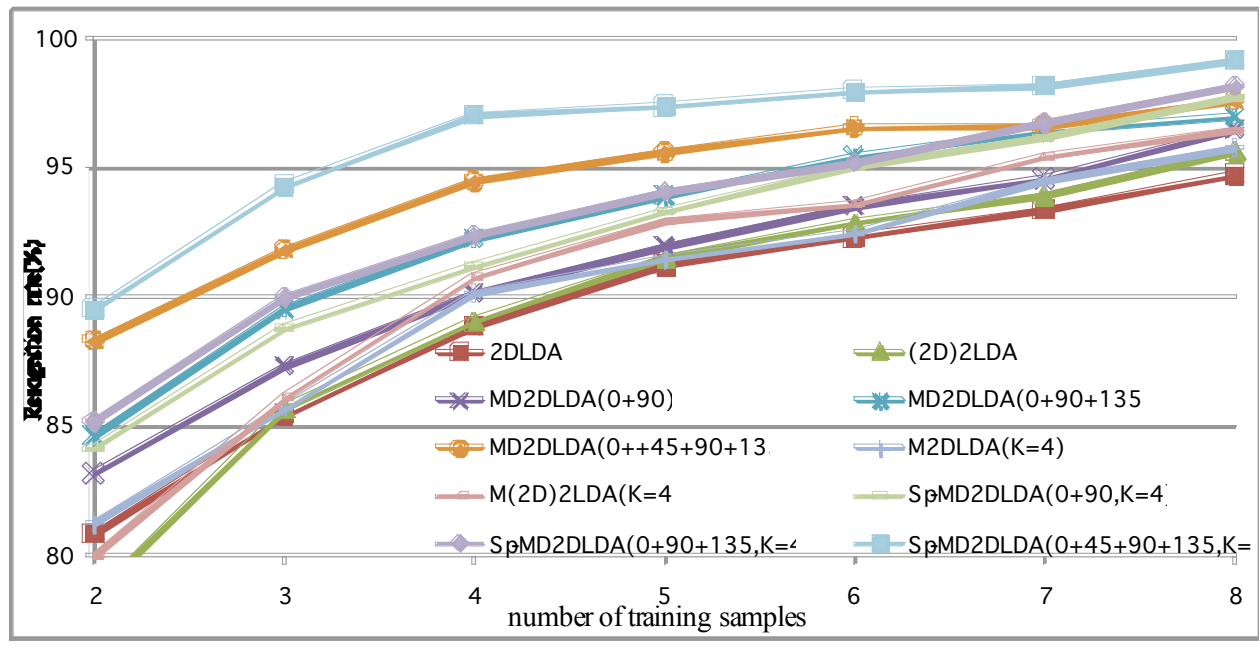

Fig. (12). Recognition rates of related methods with varying number of training samples.

The Fig. (12) shows the recognition rates of the related methods with varying number of training samples(from 2 to 8). It can be observed from Fig. (12) that the trend of recog- nition rates is up for all the methods as we increase the number of training samples. Similar to experimental results on the Yale database, from the comparison results of M2DLDA 
and 2DLDA, M(2D) $)^{2}$ LDA and (2D) ${ }^{2}$ LDA,Sp-MD2DLDA and MD2DLDA, we can also observe that the modular based methods(including M2DLDA, M(2D) ${ }^{2} \mathrm{LDA}$ and SpMD2DLDA) have higher recognition rates than the unmodular based methods(including 2DLDA, $(2 \mathrm{D})^{2} \mathrm{LDA}$ and MD2DLDA). In all the different number of training samples, the proposed Sp-MD2DLDA in the fusion directions of $0+45+90+135$ always obtains the highest recognition rates.

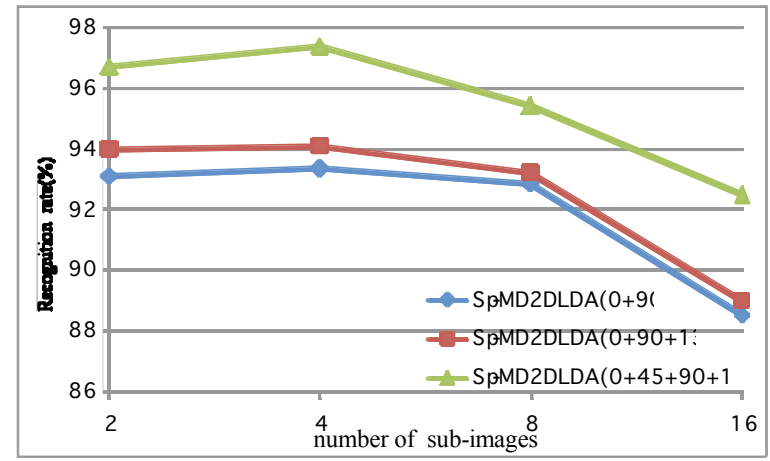

Fig. (13). Recognition rates of the proposed Sp-MD2DLDA with varying number of sub-images.

To observe the effect of different number of sub-images (denotes as $K$ ) on face recognition, we vary $K$ from $2(2 * 1)$ to $16(4 * 4)$. We can observe from Fig. (13) that the proposed Sp-MD2DLDA all have the best performance at $K=4$, and recognition rates decline when $\mathrm{k}>4$, since the global information may be destroyed by dividing the origin images into very small regions.

The comparison of the experimental results from Yale and ORL databases show that the effective directions of Yale database are 0 -degree, 30-degree, 90-degree and 150-degree, but the effective directions of ORL database are 0-degree, 45-degree, 90-degree and 135-degree. Hence, the effective directions used for feature fusion are different for different face databases. Also, in the experiments on Yale database, the proposed Sp-MD2DLDA in the case of fusion directions of $0+90+150$ and $K=8$ has the best performance. But, in the experiments on ORL database, the best performance is obtained by Sp-MD2DLDA in the case of fusion directions of $0+45+90+135$ and $K=4$. So, from all the above experiment results, we know that the most appropriate directions used for feature fusion and the number of sub-images are not the same for different databases, and that depend on the characteristics of the face databases that used to be analyzed.

\section{CONCLUSION}

A sub-pattern Multi-directional 2DLDA(Sp-MD2DLDA) for face recognition has been proposed in this paper. It has two obvious advantages as follows: first, it can extract the local features from the sample images and reduce the impact of the variations in facial expression and illumination by dividing the original images into small regions in a subpattern manner. Second, it can extract useful features in any direction which complement each other and fuse these complementary features in an effective way. Compared with the un-modular methods or the methods which extract features only in one or two directions, the proposed method is more powerful for classifying human face images.

\section{CONFLICT OF INTEREST}

The authors confirm that this article content has no conflict of interest.

\section{ACKNOWLEDGEMENTS}

This work is supported by International Science and technology cooperation projects (NO.2011DFR90720), the 2013 comprehensive reform of the professional pilot project in Guangdong Province (Z13016), the 2014 teaching reform of higher education in Guangdong Province (GDJG20142402) and the 2013 Hanshan Normal University Youth Fund Project (LQ201302). Finally, the authors would like to thank the anonymous reviewers for their constructive advice.

\section{REFERENCES}

[1] R. Chellappa, C. L. Wilson, and S. Sirohey, "Human and machine recognition of faces: A survey, "[J]. Proceedings of the IEEE, vol.83, no.5, pp. 705-741, 1995.

[2] J. Lu, K. N. Plataniotis, and A. N. Venetsanopoulos, "Face recognition using kernel direct discriminant analysis algorithms,"[J]. IEEE Trans. on Neural Networks, vol.14, no.1, pp.117- 126, 2003.

[3] K. Han, and X. Zhu, "Research On Two-Dimensional Lda For Face Recognition,"[J].Journal Of Electronics (China), vol.23, no.6, pp. 943-947, 2006.

[4] P. N. Belhumeur, J. Hespanha, D. J. Kriegman, "Eigenfaces vs.fisherfaces: recognition using class specific linear projection,"[J].IEEE Pattern Analysis and Machine Intelligence, vol.19, no.7, pp.711-720, 1997.

[5] K. ZHOU D, YANG X, PENG N S. "A modified linear discriminant analysis and its application to face recognition,"[J].Journal of Shanghai Jiaotong University, vol.39, no.4, pp. 527-530, 2005. (in Chinese)

[6] ZHUANG ZH M, ZHANG A N, LI F L. "Based on an optimized LDA algorithm for face recognition,"[J]. Journal of Electronics \& Information Technology, vol.29, no.9, pp. 2047 -2049, 2007.

[7] H. Yu, and J. Yang, "A direct LDS algorithm for high-dimensional data with application to face recognition,"[J]. Pattern Recognition, 2001,34:2067-2070.

[8] M. Li, and B. Yuan, "2D-LDA: A statistical linear discriminant analysis for image matrix". Pattern Recognition Letters, vol.26, no.5, pp. 527-532, 2005.

[9] J. Yang, D. Zhang, X. Yong, and J. Yang, "Two-dimensional discriminant transform for face recognition," Pattern Recognition, vol.38, no.7, pp.1125-1129, 2005.

[10] Y. Jin, and Q. Ruan, "Face recognition using assembled matrix distance metric based 2DLDA algorithm,"'[A]. Proceedings of 2006 8th International Conference on Signal Processing[C], 2006.

[11] S. Noushath, K. G. Hemantha, and P. Shivakumara, "(2D) ${ }^{2}$ LDA: An efficient approach for face recognition,"[J].Pattern Recognition, vol.39, pp.1396-1400, 2006.

[12] H. Du, X. Chai, F. Wang, and F. Zhang, "Face recognition using a fusion method based on bidirectional 2DLDA," Chinese Journal of Scientific Instrument, [J], vol.30, no.9, pp.1880-1885, 2009. (In Chinese)

[13] Z. Qi, and X. Yong, "Multi-directional two-dimensional PCA with matching score level fusion for face recognition,'[J]. Neural Comput \& Applic, vol.23, no.1, pp.169-174, 2013.

[14] X. Dong, and H. Chen, "Feature extraction Using a Fusion Method Based on Multi-directional two-dimensional LDA".(Under review)

[15] W. WANG, Y. YAN "LDA for face recognition based on image segmentation,"[J.] Computer Engineering and Design, vol.28, no.12, pp. 2889-2891, 2007. (In Chinese)

[16] L. Wang, J. Wu, L. Jia, "Face recognition method based on bidirectional and modular 2DLDA,"[J]. Journal of Electronic Measurment and Instrument, vol.27, no.8, pp. 760-765, 2013. (In Chinese) 
[17] S. C. Chen, and Y. L. Zhu, "Subpattern-based principal component analysis,"[J].Pattern Recognition, vol.37, no.1, pp. 1081-1083, 2004.
[18] R. Gottumukkal, and V. K. Asari, "An improved face recognition technique based on modular PCA approach,"[J]. Pattern Recognition Letters, vol.25, no.4, pp. 429-436, 2004.

Received: September 16,2014

Revised: December 23, 2014

Accepted: December 31, 2014

(C) Xiaoqing Dong; Licensee Bentham Open.

This is an open access article licensed under the terms of the Creative Commons Attribution Non-Commercial License (http://creativecommons.org/licenses/by-nc/3.0/) which permits unrestricted, non-commercial use, distribution and reproduction in any medium, provided the work is properly cited. 